\title{
EFECTOS DEL ABORTO CLANDESTINO EN LA SUBJETIVIDAD DE LAS MUJERES. RESULTADOS DE UNA INVESTIGACIÓN CUALITATIVA EN URUGUAY
}

\author{
Alejandra López Gómez y Elina Carril Berro \\ Universidad de la República -Uruguay \\ alopez@psico.edu.uy - ecarril@psico.edu.uy
}

Recibido: 27-02-2010

Aceptado: 13-04-2010

\section{Resumen}

La dimensión subjetiva implicada en el proceso de aborto voluntario en contextos de ilegalidad y penalización ha sido escasamente explorada. Cuando un aborto se produce en la clandestinidad es, por definición, un aborto inseguro, independientemente de las condiciones sanitarias en el cual éste tenga lugar. El contexto de ilegalidad y penalización se transforma en un texto que determina la experiencia y sus resultados médicos, psicológicos, familiares, sociales y jurídicos. El estudio se realizó en base a un diseño cualitativo con entrevistas en profundidad a mujeres que abortaron clandestinamente en los últimos 15 años en Uruguay. Los hallazgos permitieron conocer los factores subjetivos asociados a las experiencias de aborto en un contexto, como el uruguayo, donde la práctica es tipificada como delito por la ley vigente en el país.

Palabras claves: aborto clandestino, subjetividad, derechos de las mujeres.

\begin{abstract}
The subjective dimension involved in the process of voluntary abortion in contexts of illegality and criminalization hasn't been studied in depth. A clandestine abortion is, by definition, an unsafe abortion, regardless of the sanitary conditions under which it takes
\end{abstract}


place. The context of illegality and criminalization turns into a text that determines the experience and the medical, psychological, family, social and legal outcome. The study was carried out on the basis of a qualitative design with in-depth interviews to women who underwent clandestine abortions in the past 15 years in Uruguay. The findings allowed for the discovery of the subjective factors associated to abortion experiences in a context, such as the Uruguayan, where the practice is defined as a crime by the law in force.

Key words: clandestine abortion, subjectivity, women's rights.

\section{Introducción}

Los contextos legales que penalizan total o parcialmente la práctica del aborto, no inhiben que las mujeres acudan a esta solución cuando se enfrentan a un embarazo que no desean o no pueden continuar. En países como Uruguay, donde el aborto es considerado un delito por la legislación vigente (Ley 9763, de 1938), se ha desarrollado un sistema clandestino que ofrece servicios de diferente calidad y confiabilidad técnica, tipo de procedimiento y costo económico, los cuales tienen como denominador común su actuación al margen de la ley.

Toda práctica de aborto que se produzca en condiciones de clandestinidad puede ser definida como aborto inseguro, ya que la salud de las mujeres está expuesta a distinto tipo de riesgos (sanitarios, subjetivos, legales, sociales) de los cuales, generalmente, no se hacen cargo quienes realizan los procedimientos (OMS, 2003).

Cuando se considera el aborto inseguro como un problema de salud pública pocas veces se repara en el impacto psicológico que tiene esta práctica producto de su realización en contextos de ilegalidad y clandestinidad. Las marcas en la subjetividad que deja la experiencia del aborto clandestino y el aborto inseguro han sido escasamente exploradas por quienes investigan en este campo. La literatura científica disponible 
alerta sobre los efectos sanitarios negativos del aborto inseguro. Mucho se ha escrito también sobre las consecuencias socio-legales de esta práctica para las mujeres. Sin embargo, poco se conoce acerca del impacto en sus subjetividades.

La revisión sobre aspectos psicológicos del aborto voluntario en la región de América Latina confirma que existe escasa investigación sobre el tema y que los artículos de divulgación disponibles no se basan, habitualmente, en evidencias científicas. A nivel internacional, existe un mayor volumen de reportes publicados. En tal sentido, las recientes revisiones sistemáticas realizadas por Brenda Major y colaboradoras/es ofrecen sólidas reflexiones sobre la vinculación entre aborto y salud mental (Major et al., 2009), las cuales actualizan el reporte realizado por la American Psychological Association (APA) Task Force on Mental Health and Abortion (TFMHA), realizadas en 2008 (APA, 2008). Algunas investigaciones indican resultados positivos muy probables cuándo las mujeres pueden hacer las elecciones informadas, basadas en sus propios valores (Stotland, 2003). Otras afirman que se podría manifestar en las mujeres algún síntoma de depresión (Nordal et al., 2005a; 2005b). La mayoría rechazan la existencia de efectos traumáticos o la presencia de un síndrome traumático post aborto (SPTA) (Knowles, 2007; Fielding y Schaff, 2004; Faúndes y Barzellato, 2005; Amuschástegui, 2005). Para que el STPA se manifieste sería necesario que las personas estuvieran sometidas a eventos traumáticos inesperados e incontrolables y sientan amenazada su integridad física (Lee y Gilchrist, 1997; Gómez Lavin y Zapata García, 2005).

La mayoría de las investigaciones refieren que la realización de un aborto voluntario no es causa suficiente para la posterior aparición de problemas en la salud mental en las mujeres. Toman en cuenta los factores psicológicos pre-existentes en la persona que podrían o no favorecer la aparición de trastornos psíquicos posteriores. Las que sostienen que el aborto es causante de complicaciones psicológicas severas, señalan que éstas pueden estar asociadas a la existencia de factores predisponentes, lo cual implicaría la importancia de determinar su presencia previo a la realización de un aborto (Reardon, 2003 y 2006). 
Los estudios señalan factores de riesgo que podrían favorecer la emergencia de trastornos psicológicos en el post aborto. Entre ellos, la relación entre depresión y contexto socio legal donde tiene lugar la práctica (Lacerda y Ribero, 2003; Lee y Gilchrist, 1997); la existencia de enfermedades psiquiátricas o estado de la salud mental previa a la realización del aborto (Major et al., 2000; Stotland, 2003); la presencia de problemas emocionales no resueltos (Coleman et al., 2005), la exposición a la sanción social (Lacerda y Ribero, 2000), la ausencia o debilidad de apoyo social y familiar (Lee y Gilchrist, 1997; Reardon, 2003); la presión ejercida por personas significativas para la mujer (Lacerda y Ribero, 2000); el lugar de la pareja (o el co-genitor) en la toma de decisión (Adler et al., 1990; Cohen, 2006; Pope, Adler y Taschann, 2001; Nordal et al., 2005a y 2005b).

Un hallazgo relevante aportado por algunas investigaciones refiere a que el momento de mayor angustia psicológica para una mujer se ubica en el período anterior al aborto (Major et al., 2000; Major, 2003; Cohen, 2006; Pope, Adler y Taschann, 2001; Lee, Gilchrist, 1997). Mientras que el alivio es el sentimiento predominante luego de su realización (Adler et al., 1990; Adler, 2000). El análisis de 14 estudios realizados en los Estados Unidos, muestra que la mayoría de las mujeres (76\%) reportaron sentir alivio y que si el aborto fue seguro y sin presiones externas, se encontró escasa patología en el período posterior. Mujeres con creencias religiosas muy fuertes, que viven en países donde las leyes son muy restrictivas y/o en sociedades donde están expuestas a una gran presión social, pueden sufrir mayores consecuencias psicológicas adversas (Langer y Glas, 2003).

El presente artículo se basa en los resultados del estudio "Aspectos psicológicos del aborto voluntario en contextos de ilegalidad y penalización. Significados, prácticas y efectos en mujeres uruguayas" (Carril y López Gómez, 2008), el cual fue implementado por las autoras en el marco de su trabajo de investigación en la Cátedra Libre en Salud Reproductiva, Sexualidad y Género de la Facultad de Psicología de la Universidad de la República (UR). Contó con el apoyo de la Comisión Sectorial de Investigación Científica (CSIC) de la UR en calidad de proyecto I + D (2005-2007). 
El estudio tuvo como principales objetivos conocer los significados asociados a las prácticas de aborto voluntario para las mujeres a partir de sus experiencias personales; identificar la presencia de efectos psicológicos y su relación con las condiciones y circunstancias en las que se desarrolló la práctica; analizar las rutas y procesos de decisión y su relación con la existencia de posibles efectos psicológicos y caracterizar los aspectos psicológicos implicados en el aborto voluntario.

\section{Metodología}

La investigación cualitativa con su énfasis en abordar los estudios en profundidad a partir de un número limitado de casos, fue considerada la más adecuada para identificar y comprender los aspectos subjetivos y posibles efectos psicológicos de las prácticas de aborto voluntario.

Se diseñó un estudio cualitativo mediante el uso de la técnica de entrevista en profundidad con mujeres que transitaron por la experiencia de aborto voluntario. El uso de esta técnica permitió acceder a una descripción densa y profunda de los aspectos más relevantes a estudiar. La entrevista en profundidad acompañada de una escucha técnica psicológica, permitió identificar aspectos psicopatológicos no imputables a la situación de aborto, pudiendo distinguir los aspectos subjetivos involucrados de aquellos posibles efectos psicológicos traumáticos.

Se previó la aplicación de técnicas de diagnostico psicológico en los casos donde en la entrevista se observaran elementos relacionados con trastornos de personalidad, antecedentes depresivos severos y/o intentos de autoeliminación, consumo de sustancias psicoactivas, alcoholismo, antecedentes psiquiátricos (u otros elementos psicopatológicos significativos), con el objetivo de precisar aspectos de la personalidad que pudieran ser previos a la situación de aborto._De esta forma se consideró que la investigación lograba una mejor aproximación a la construcción del dato. 
Se diseñó una muestra intencional teórica que incluyó a mujeres de edades entre 20 y 50 años, que hubieran vivido la experiencia personal del aborto voluntario en los últimos 15 años y que pertenecieran a niveles educativos diferentes. El nivel educativo fue considerado en función de los años de instrucción (bajo: hasta seis años; medio: de 7 a 12 años y alto: 13 años y más). El plazo de los 15 años fue a los efectos de contemplar un criterio de temporalidad que permitiera considerar comparativamente los posibles efectos psicológicos inmediatos y mediatos de la experiencia. Se buscó incluir a mujeres con y sin hijos. Se incluyó a mujeres que hubieran vivido, por lo menos, una experiencia de aborto voluntario. En los casos de mujeres con más de un aborto, se procedió a explorar específicamente la última experiencia vivida, si bien no se descartó la información aportada espontáneamente por las mujeres respecto a sus otras experiencias. La inclusión de casos quedó supeditada al criterio de saturación de la información y de heterogeneidad de la muestra en función de los criterios muestrales definidos.

Las entrevistas fueron realizadas en lugar acordado con las mujeres. Algunas optaron por sus propias casas y otras, en el lugar ofrecido por las investigadoras. En todos los casos se procuró generar un clima de reserva e intimidad de modo de preservar la confidencialidad. El tiempo de duración de cada entrevista fue de aproximadamente una hora y media. Se realizó una ficha individual para relevar datos sociodemográficos básicos, las cuales fueron sistematizadas para su utilización en el análisis del material.

Se diseñó una pauta de entrevista con preguntas abiertas para conocer los factores psicológicos mencionados en forma espontánea y se incluyeron preguntas dirigidas sobre factores identificados en otros estudios, para comprobar su presencia en nuestra población. 


\section{Análisis de la información}

Las entrevistas fueron grabadas para su posterior transcripción, codificación, sistematización y análisis. El procesamiento y análisis de la información se realizó en base a códigos elaborados a partir de la pauta de entrevista y se incluyeron temas emergentes que surgieron durante las sesiones. La codificación del material de campo se realizó con la participación de dos integrantes del equipo de investigación como estrategia de triangulación metodológica mediante la cual se pudieron resolver discrepancias iniciales en el proceso de codificación y sistematización.

Para la codificación de los grandes temas se procedió de manera deductiva, mientras que en la codificación fina que se realizó con las sucesivas lecturas del material, se procedió de manera inductiva. Se trató de identificar lo que se dice sin decir, los implícitos y supuestos (teóricos, ideológicos) que organizan el discurso de los participantes.

El marco conceptual desde el cual se analizó el material se basó en herramientas teóricas de los Estudios de Género aplicados al campo de la salud sexual y reproductiva junto con aquellas que provienen del campo psicoanalítico para la comprensión de las subjetividades implicadas. Para favorecer el tratamiento de la información se utilizó el paquete informático Ethnograph V 5.0.

Se realizó un análisis temático de cada entrevista (análisis longitudinal) para luego proceder a un análisis transversal del material recogido, de modo de establecer comparaciones entre los discursos de las entrevistadas, pudiendo identificar los denominadores comunes y las diferencias significativas. 


\section{Consideraciones éticas}

Se entrevistó a aquellas mujeres que informada y voluntariamente accedieron a la situación de entrevista, conociendo previamente los objetivos, beneficios y resultados esperados de la investigación, garantizando la protección del anonimato de la persona, mediante el cuidado de su no identificación. No se solicitó consentimiento firmado a las mujeres, entre otras razones, por la situación de ilegalidad y penalización del aborto en el país.

En concordancia con el principio de justicia, consideramos que la investigación debía contar para aquellas mujeres que lo requiriesen o que lo demandasen, un adecuado sistema de referencia y derivación a espacios donde las mujeres pudieran elaborar y procesar la situación. En aquellos casos en los cuales se detectasen trastornos de personalidad y/o síntomas de entidad -tal como se mencionó anteriormente- se había previsto la realización de entrevistas para la devolución de los resultados del estudio y realizar posibles orientaciones psicoterapéuticas.

\section{Resultados}

Se entrevistó un total de 25 mujeres con edades entre 19 y 69 años. Todas ellas habían tomado la decisión voluntaria de interrumpir un embarazo. Cinco pertenecían a nivel educativo bajo, nueve al nivel medio y once al nivel alto. La mayoría tenían trabajo remunerado fuera de la casa, mientras que tres se dedicaban exclusivamente al trabajo doméstico de la casa. Al momento de la entrevista, nueve tenían pareja conviviente, cinco tenían pareja no conviviente y once no estaban en pareja. En cuanto a las creencias religiosas, cinco se definieron como católicas creyentes, 14 manifestaron no creer en la existencia de ningún dios y seis se definieron como católicas no practicantes. 
Respecto a la edad en la que ocurrió el aborto, 13 tenían menos de 25 años, siete tenían entre 26 y 35 años y cinco tenían más de 36 años. Del total de mujeres, 11 eran madres al momento del aborto y 14 no lo eran. En cuanto a la distancia temporal con la experiencia, 12 se habían practicado el aborto (o el último aborto) hasta seis meses antes de la entrevista mientras que 13 se habían practicado el aborto a un año y más de la fecha de la entrevista. Dos mujeres se lo habían practicado más de 15 años antes de la entrevista. Se valoró como pertinente incluir estos casos que estaban fuera de los criterios muestrales iniciales, a los efectos de analizar el pasaje del tiempo en la elaboración de la experiencia. Del total de mujeres, 17 se habían practicado un aborto y siete se habían realizado entre dos y tres. Una mujer se había realizado cuatro abortos. En cuanto al tipo de procedimiento utilizado, 12 lo hicieron mediante técnicas quirúrgicas (AMEU o legrado) y 13 mediante medicamento (misoprostol). Ninguna de las mujeres entrevistadas tuvo complicaciones o secuelas médicas. En todos los casos, salvo una mujer, el aborto tuvo lugar antes de las 12 semanas.

La información recogida en las entrevistas fue organizada en función de seis categorías: a) las mujeres frente al embarazo no esperado, b) las mujeres frente a la decisión, c) las mujeres frente a la práctica, d) efectos psicológicos del proceso de aborto voluntario, e) opiniones sobre aborto en Uruguay y f) los varones en el discurso de las mujeres.

\subsection{Las mujeres frente al embarazo no esperado}

Las reacciones de las entrevistadas frente a la constatación del embarazo fueron de sorpresa, estupor, indignación y miedo. Estas reacciones no estuvieron relacionadas con la edad, el tener o no hijos o el nivel educativo. El embarazo fue significado como un hecho inesperado, concientemente no buscado y, en un principio, sin poder comprender cómo había sucedido. No podían dar sentido a un embarazo que no buscaron, que no querían continuar y cuya metáfora fue, muchas veces, la muerte. Ese embarazo irrumpió en la existencia de las mujeres y generó montos de ansiedad 
importantes, desorganizó el proceso de pensamiento y puso en marcha mecanismos psíquicos de defensa: negación, renegación y regresión. Estos embarazos inesperados, jaquearon la omnipotencia, la certeza en las previsiones y la fantasía de dominar el cuerpo.

"En el momento que me pasó, casi me muero, casi me muero. La verdad, fue espantoso para mí. Era sacármelo o morirme. No quería, no quería tenerlo. Para mí era algo espantoso. Me sentía tan indefensa, saber que yo no podía hacer nada, me hice tres tests de embarazo, me gasté toda la plata que tenía en el momento haciéndome tests porque no podía creerlo" (Paulina, 24, NEM).

Las mujeres se enfrentaron a la posibilidad de una maternidad no deseada, en su dimensión simbólica, cultural y social. Lo hicieron a partir de sus vivencias, sus experiencias vitales y momento de vida, su grado de autonomía y los sentidos y representaciones que tenía la maternidad para ellas. Los significados asociados al aborto no pueden desligarse de aquellos vinculados a la maternidad así como al contexto legal y social en el cual éste tiene lugar.

La mayoría de las entrevistadas conocía y había usado métodos anticonceptivos, en particular preservativo masculino, anticonceptivos orales, anticoncepción de emergencia y DIU. Hubo quienes utilizaban el método de ritmo. Otras habían solicitado en sus servicios de salud, acceder al procedimiento de la esterilización. Pero no habían tenido éxito, ya que este método anticonceptivo no está garantizado para todas las mujeres. Algunas pocas no habían utilizado método anticonceptivo alguno, argumentando que les resultaba muy difícil la negociación de su uso con sus parejas o compañeros sexuales. El relato de las mujeres dejó en evidencia la ambivalencia respecto al autocuidado, la no infalibilidad de los métodos anticonceptivos, los factores subjetivos asociado a su uso incorrecto y las relaciones de poder que determinan modalidades desiguales de negociación sexual. 
"Me dio rabia conmigo misma, yo sentí mucha rabia por mi descuido, por mi desorden, que no aprendas [...]” (María, 47, NEA).

Las mujeres opinaron sobre la maternidad y expresaron sus sentimientos sobre las prácticas maternales y los significados sociales a ellas asociadas. Se pudo observar que los discursos diferían según el nivel educativo. Las mujeres con niveles educativos medios y altos, que contaron con posibilidades de desarrollo personal y mayor grado de autonomía no concebían a la maternidad como la única fuente de realización y en algunos casos, dudaban si querían ser madres.

"No, porque no lo siento. No es lo que quiero para el presente y el futuro. No me veo como madre" (Patricia, 26, NEM).

"Me encantan los niños, me encantan los bebes vivo rodeada de bebés. Pero, no tengo paciencia para madre. No sirvo" (Carolina, 24, NEM).

Las mujeres de nivel educativo bajo fueron quienes identificaron la maternidad con sacrificio, entrega y destino de vida. Para algunas, como un mandato de Dios, un designio divino que trasciende la voluntad individual.

"Porque a mí no me gusta abortar hijos. Si vienen, por algo vienen. Estos son mis hijos [se los presenta a la entrevistadora] dan trabajo, pero son mis adoraciones, mis hijos son mis ojos y mi vida a la vez" (Melina, 35, NEB).

Para algunas mujeres la maternidad como destino coexistía con la maternidad como opción. Fue en ellas donde la idea de responsabilidad frente a un hijo apareció con más fuerza: el cuidado no sólo fue entendido en términos de alimentación y atención de la salud, sino de transmisión de valores y sostén afectivo. Estas mujeres sentían no estar preparadas, implicándoles además una negociación con otros deseos personales y con las exigencias del mundo laboral/profesional. 
Aquellas mujeres que experimentaron sentimientos ambivalentes frente al embarazo, vivieron una lucha interna entre un aspecto de sí mismas que no quería $-\mathrm{o}$ no podía- y otro que la condenaba por el no deseo. Al decidir abortar, lo hicieron desde la duda lo cual se tradujo luego en un intenso malestar subjetivo.

"No estaba totalmente segura, es más, estuve en duda hasta el último momento, hasta que estaba en la camilla. Era confuso, estaba angustiada. No sabía si practicarme el aborto o no, fue una indecisión hasta último momento. Una parte de mi quería practicarse el aborto. Es que son reacciones encontradas, porque una siente que está bajo una presión terrible, una inseguridad terrible. No quería ese embarazo. Sentí muchas reacciones, diferentes sensaciones, sentimientos [...] (Alma, 41, NEA).

En mujeres con nivel educativo bajo, la ambivalencia y la duda se manifestaron como la lucha entre sostener un interés personal y la significación imaginaria del aborto como un asesinato, lo que abrió el camino a una representación de sí mismas como asesinas, filicidas, emergiendo de este conflicto, sentimientos de angustia, culpa y autoreproches. Pero también surgió el miedo por el aborto, por el temor a perder la vida en una práctica que, por las condiciones inseguras en que tenía lugar, el riesgo de vida siempre es posible.

\subsection{Las mujeres frente a la decisión}

Se indagó sobre tres tipos de recursos para la toma de la decisión frente a un embarazo no esperado: información, sostén afectivo y social y disponibilidad de dinero. Estos recursos se tornan especialmente importantes cuando se trata de decidir sobre un aborto y ejecutar esa decisión en contextos de ilegalidad y penalización. 


\section{$\underline{\text { Recursos de información }}$}

El acceso a información confiable sobre cómo es una práctica segura de aborto y qué se requiere para efectivizarla, dónde acudir, los tipos de práctica existentes, los riesgos de una práctica insegura, son elementos de gran importancia para la toma de la decisión frente a un embarazo que no se desea continuar. La dificultad para acceder a este tipo de información fue un elemento generador de altos montos de ansiedad y angustia en las mujeres. La clandestinidad determina que la información no sea de fácil acceso. Una vez tomada la decisión, era sustantivo poder resolver la práctica en el menor plazo posible, porque cuanto antes se produjera el aborto, menores iban a ser los cambios en el cuerpo y menores las posibilidades de "sentirse" embarazadas.

Se observó que muchas mujeres no disponían a priori de información alguna y ésta fue proporcionada por personas de sus entornos cercanos. Esta situación estuvo relacionada con la falta o escasa disponibilidad de información sobre sexualidad y reproducción, las débiles condiciones sociales para el ejercicio de los derechos reproductivos, la fantasía sobre la invulnerabilidad personal que se traduciría en la frase "no me va a pasar a mi” y el contexto de ilegalidad que genera que la práctica del aborto continúe siendo predominantemente una práctica privada, a pesar de los avances notables que se pueden señalar en el campo del debate social en los últimos años.

La necesidad de contar con información de calidad (técnica, humana) fue referida por las mujeres en relación a dos aspectos: el proceso del aborto (cómo es, qué se siente en el cuerpo, qué va a suceder) y el método y lugar más seguro para realizarlo. El temor al daño, la angustia por no saber o el tener información fragmentada y/o parcial fueron algunos de los factores que acompañaron el proceso de toma de decisión en algunas mujeres, generando una cierta paralización frente a la situación.

"No tenía a nadie que me informara, no tenía absolutamente a nadie. Lo que pensaba era que no lo quería tener, pero no pensaba en ir a una clínica a hacerme un aborto. [...] Ir a una clínica, cuando las clínicas 
lamentablemente... son malas, están haciendo las cosas mal, por más que vos estés pagando muchísima plata [...]" (Sara, 24, NEB).

Varias mujeres hicieron referencia al complejo recorrido que supuso el acceso a información confiable. La existencia de la Normativa Proteger la vida y la salud de las mujeres mediante la prevención del aborto provocado en condiciones de riesgo (Ministerio de Salud Pública, Ordenanza nº 369, 08/2004) debería marcar una diferencia sustantiva, en la medida que esta norma reconoce el derecho de toda mujer en situación de embarazo no deseado de acceder a información y orientación sobre las alternativas que dispone para tomar una decisión. La no implementación de la normativa en todos los servicios de salud del país y su no difusión amplia en la población, genera que este recurso sea poco conocido por las mujeres.

Las rutas de acceso a la información implicaron un recorrido complejo, sostenido fundamentalmente en el apoyo de personas del entorno inmediato o de otras con quienes se tenía un vínculo intermediado por un familiar o amistad.

"Empezamos con la búsqueda de un lugar. Era enero, pleno verano. Mi madre tenía un conocido, lo llamamos y no atendía el teléfono. Yo conseguí dos teléfonos más por unas amigas y no existía más esa clínica. Al final, responde al llamado una persona conocida de mi madre. Fue la salvación" (Amelia, 30, NEA).

\section{$\underline{\text { Recursos de apoyo afectivo }}$}

La presencia o ausencia de personas significativas para las mujeres como sostén, fue uno de los aspectos referidos en las entrevistas. La gran mayoría de las mujeres relataron haber contado con el apoyo y la solidaridad de personas de su entorno, quienes jugaron un rol importante para que las mujeres pudieran transitar por este proceso con respaldo y acompañamiento. Pero no todas encontraron respaldo. Hubo quienes sintieron rechazo, censura, aislamiento. Para ellas, toda la experiencia fue más penosa. Hubo un denominador común en todas las mujeres, con distinta intensidad y 
significación, refirieron haber vivido expresiones de sanción social y cuestionamiento moral por la decisión tomada.

Esas personas significativas fueron fundamentalmente otras mujeres: madres, amigas, tías, abuelas, compañeras de trabajo, cuñadas, suegras. La actitud de esas otras mujeres fue de identificación con el malestar / ansiedad / sufrimiento / desesperación / perplejidad que vivieron las entrevistadas, si bien sus respuestas no fueron homogéneas. De todos estos recursos de apoyo, las madres ocuparon un lugar de particular importancia.

"El día que me hice el análisis de sangre se lo dije a mi mamá y a mi hermana. El hecho de haber quedado embarazada era una frustración. Pero ellas me apoyaron y me dijeron que iban a estar al lado mío" (Leticia19, NEA).

"Fui a hablar con una de mis tías, porque yo no tengo madre. Es como mi madre postiza. Mi madre falleció cuando yo era niña. Y entonces me dijo que la decisión era mía [...]” (Noelia, 22, NEM).

Las madres de las entrevistadas, en su presencia o en su ausencia, fueron denominador común en el relato de las mujeres. La intensidad e importancia de la relación madre-hija quedó en evidencia. Los relatos sobre la experiencia del aborto, pusieron de manifiesto el tipo de relación que tenían o habían tenido con sus madres, en un gradiente que fue desde el reclamo al reconocimiento y la gratitud. Algunas mujeres, invirtiendo la relación de cuidado, ocultaron el hecho a sus madres, para evitar angustiarlas.

En el caso de los varones, co-genitores, parejas, padres, hermanos, amigos, su lugar estuvo asociado al sostén económico. En lo que se refiere al apoyo afectivo, aún en aquellas mujeres cuya decisión fue compartida y acompañada por el co- genitor y/o pareja, refirieron a otras personas como los puntos de apoyo afectivo y práctico. Este se tradujo en compañía, en posibilidad de compartir dudas y miedos, en mayor tranquilidad, en apoyo logístico y en barrera para la exposición al riesgo. La situación 
de aborto provocó en las mujeres, la puesta en marcha de mecanismos de regresión, expresado en el impulso de volver hacia atrás en el tiempo, el sentirse como niñas, necesitadas de protección y cuidado.

En el caso de las mujeres jóvenes que no contaban con apoyo familiar o temían no tenerlo, ese lugar fue ocupado por sus pares, las amigas basadas en la empatía, identificación y complicidad generacional.

La clandestinidad de la práctica impacta emocionalmente en las mujeres, especialmente cuando no disponen de apoyo afectivo. El temor al rechazo sociofamiliar y a la sanción legal generó en algunas de las entrevistadas, el autosilenciamiento, como forma de preservar la decisión, de no exposición al daño emocional y de evitar conflictos con seres queridos. Sin embargo, el efecto fue de similares características al que se buscaba evitar.

"Creo que es lo peor que uno carga. Además de pasar por el aborto, el tema de la ilegalidad, que hace que tengas que estar con mucho más cuidado. Cuidar que nadie lo sepa, que nadie opine y además por supuesto, no podés decir, en cada lugar, lo que estás viviendo. Eso emocionalmente hace daño porque solamente una sabe por lo que estás pasando, sin poder comunicarlo libremente hacia los demás. Eso es lo peor que tiene la situación... es lo más traumático que tiene la situación" (Lidia, 25, NEA).

\section{Disponibilidad de recursos económicos}

Una vez tomada la decisión, el paso siguiente es resolver el acceso a la práctica del aborto. En contextos donde la práctica es ilegal, el acceso se transforma en una de las principales preocupaciones. Además de contar con recursos de información confiable y apoyo afectivo, las mujeres necesitaron resolver la disponibilidad de recursos económicos. Hubo una correlación entre tener apoyo afectivo y acceso a recursos económicos. En el caso de las mujeres jóvenes, los padres u otros familiares directos jugaron un papel central en brindar este soporte. En otras, la participación del 
co-genitor, pareja u otros varones del entorno cercano estuvo limitada, a apoyar con dinero. Este rol tradicional del modelo hegemónico masculino operó también en estas circunstancias. Otras mujeres resolvieron solas, sin apoyo de terceros: acudieron a préstamos bancarios, solicitaron adelantos de salario o se ajustaron en los gastos durante unos meses. La motivación para interrumpir el embarazo era tan fuerte que, aún contando con ingresos económicos limitados, aún comprometiendo su presupuesto cotidiano o endeudándose, no desistieron de su decisión. Esta situación se tornó especialmente dramática en mujeres de escasos recursos económicos. Así como el embarazo fue vivido como un acontecimiento no previsto, tener el dinero para interrumpirlo tampoco estaba en sus planes. Sin embargo, todas desarrollaron estrategias para acceder al aborto. Esta es una de las razones por las cuales estas mujeres tuvieron prácticas "más seguras".

\subsection{Las mujeres frente a la práctica de aborto}

Ninguna de las mujeres entrevistadas se había practicado un aborto de riesgo y/o había padecido complicaciones médicas severas. Sin embargo, el relato de las mujeres fue contundente respecto a la desprotección y exposición al daño emocional.

El tipo de procedimiento utilizado por las mujeres fue el uso de medicamento (misoprostol) y técnicas quirúrgicas (legrado o AMEU). Ambos procedimientos son considerados seguros y eficaces, siempre y cuando sean realizados por personal entrenado y en el marco de servicios de salud mediante una atención integral a lo largo de todo el proceso, lo cual incluye adecuada orientación, seguimiento y acompañamiento técnico.

La presencia de ambos tipos de práctica estuvo asociada a la distancia temporal entre el momento de realización de las entrevistas y el del aborto. Quienes habían abortado luego del 2005, lo habían hecho mediante el uso de misoprostol confirmando la creciente incidencia de este método en los últimos años en el país y en la región de Latinoamérica asociado a la difusión de información sobre la eficacia del método, su 
menor costo económico y las mejores posibilidades de acceso en el mercado clandestino. Su realización de manera ambulatoria o en la casa de la mujer, las posibilidades de ser auto administrado y la no necesidad de anestesia general, lo ubican como un método "más seguro" y más viable para la práctica de aborto en contextos de ilegalidad y penalización.

Las mujeres cuyas prácticas de aborto fueron anteriores al 2005, lo hicieron mediante métodos quirúrgicos realizados en clínicas clandestinas con mayores o menores garantías de seguridad en el procedimiento y sus resultados médicos.

\section{Experiencias de aborto quirúrgico}

La mitad de las mujeres entrevistadas había acudido a clínicas clandestinas. Las rutas de acceso, el tipo y calidad de atención, el método utilizado, los costos y los resultados fueron diversos, en estrecha vinculación con el poder adquisitivo de las mujeres y con el momento histórico en el cual se realizó el procedimiento. Aquellas cuyos abortos habían tenido lugar en los últimos cinco años encontraron mayores dificultades para acceder a clínicas clandestinas con mejores garantías. Como producto de la represión policial, éstas cambiaron su ubicación o habían dejado de funcionar. Esta situación provocó altos montos de ansiedad y preocupación en las mujeres, quienes eran conscientes que disponían de poco tiempo para efectivizar su decisión. Una vez ubicada la clínica, luego de concertar telefónicamente una cita, se iniciaba otro proceso generador de incertidumbre y ansiedad. Todas las mujeres relataron que la información que recibieron inicialmente era mínima. El lugar de la entrevista previa no sería el mismo lugar donde luego se realizaría el procedimiento. En esta instancia se les proveía de mayor información sobre el tipo de método, indicaciones médicas previas, costos del procedimiento y condiciones. Allí se acordaba un día y hora para que las mujeres se presentaran en un punto de la ciudad, al cual no podían ir acompañadas. Se las recogía en un auto y se las trasladaba, luego de dar un largo rodeo por la ciudad, a un local en el cual se realizaría la interrupción. Estos locales aparentaban ser casas particulares ambientadas para funcionar como clínicas. 
"La misma muchacha que me atendió la primera vez, me pasó a buscar por XX [se refiere a una esquina de Montevideo], me pasaron a buscar por ahí, nos llevaron a otro lado. No podes ir con nadie. Permitían solamente creo que fuera la madre si la chica era menor de edad, que me enteré porque pasamos a buscar a otra chica que era menor, que al final no pudo ir porque no había respetado el ayuno. Había, no te quiero mentir, pero debería haber unas 8 mujeres más o menos [...]" (Carolina, 25, NEM).

"Era una casa en realidad, además fuimos ahí, esperamos un poquito, porque éramos las primeras, porque después cuando salimos había más gente, pero cuando llegamos había poquita gente. Prendieron una estufa a leña y después nos preparamos, nos llevaron a un cuartito con dos camas [...]" (Patricia, 24, NEM).

Las mujeres debían "confiar" en la palabra del personal de la clínica que las entrevistaba. Al no poder ser acompañadas, el temor al daño, la preocupación por los riesgos, el sentimiento de desprotección y de vulnerabilidad se incrementaba notablemente. Las mujeres lo único que querían era que "todo terminara pronto".

La experiencia de aquellas mujeres que abortaron en clínicas clandestinas hace más de cinco años, tuvo matices respecto a aquellas que lo habían hecho más recientemente. Si bien el aborto era ilegal, el acceso a las clínicas era más directo: se concertaba cita por teléfono, se mantenía una entrevista, se conocía el lugar y podían estar acompañadas. La intensificación de los controles hacia las clínicas como consecuencia de la represión policial, tuvo consecuencias en el acceso. Otra diferencia fue el costo del procedimiento, el cual osciló entre 400 y 800 dólares americanos, siendo éste un factor de angustia para las mujeres, quienes debieron desarrollar estrategias para obtener el dinero.

En cuanto al tipo de método utilizado, todas las mujeres recibieron anestesia general, pero no todas pudieron precisar si el procedimiento fue mediante legrado o 
aspiración endouterina. Esta dificultad para dar cuenta del tipo de método, está probablemente asociado a la calidad de la información recibida y a la baja relevancia que para las mujeres tenía ese dato en aquel momento. Lo que más les preocupaba era saber si el procedimiento era seguro y eficaz.

Las mujeres relataron que les impactó encontrar salas de espera "llenas de mujeres esperando para ser atendidas". En ese momento, fue un alivio saber que había otras en su misma situación y que había testigos, si algo no "andaba bien”. Encontrarse con ese panorama, les permitió a algunas tomar conciencia de la magnitud de la práctica en el país.

Las experiencias fueron recordadas con intensidad y detalle, independientemente del tiempo transcurrido al momento de la entrevista y dieron cuenta del registro en el cuerpo: un malestar inmediato que, con el paso de las horas se fue tornando difuso hasta desaparecer. Algunas ubicaron el malestar en los efectos de la anestesia, otras en el "dolor en el útero". Esta es una diferencia notable con el aborto con medicamento, donde la corporeidad está presente de manera intensa durante todo el proceso del aborto y no solamente en el post evento.

Las clínicas clandestinas no garantizan calidad y calidez en la atención. Pueden ofrecer mayores o menores grados de seguridad en los procedimientos y eficacia en los resultados. La calidad de atención recibida por las mujeres incluyó experiencias positivas respecto a la buena atención, si bien la situación de la clandestinidad es por definición violenta. En todos los casos, las mujeres recibieron indicaciones post evento para evitar complicaciones, incluyendo tratamiento antibiótico, así como información respecto a sintomatología esperable. En ningún caso se presentaron complicaciones severas. 


\section{Experiencias de aborto con misoprostol}

Del total de mujeres entrevistadas, trece de ellas accedieron a un aborto con medicamento. Estas experiencias se remontaban a los últimos años, seguramente asociado a una difusión más amplia del misoprostol en Uruguay. En contextos de ilegalidad y penalización, el uso del misoprostol ofrece ventajas comparativas con el aborto quirúrgico, en términos de seguridad y autonomía en las mujeres. El costo de la medicación en el mercado clandestino es relativamente menos oneroso que un aborto quirúrgico médicamente seguro. El misoprostol es de venta controlada en farmacias, bajo receta profesional de un médico gastroenterólogo, estando prohibida su venta para uso ginecológico. Fue habilitada su utilización intra - hospitalaria con fines obstétricos en el año 2005, por el Ministerio de Salud Pública. Ello incluye tratamiento de aborto incompleto, inducción del trabajo de parto y maduración cervical.

Las mujeres del estudio accedieron a información sobre este método fundamentalmente a través del asesoramiento que algunos servicios públicos de salud y /o gineco-obstetras realizan a mujeres en situación de embarazo no deseado, en el marco de la normativa sanitaria ya mencionada (MSP, ordenanza $N^{o} 369$ ). Otras lo hicieron a través de internet y otras a través de mujeres que habían usado este método. Otro capítulo lo constituye el acceso a la medicación. Esto implicó no sólo disponer del dinero para la compra, sino ingresar a los circuitos clandestinos de venta. Algunas mujeres se comunicaron con farmacias para saber el costo y los requisitos de compra. Las respuestas que encontraron fueron diversas en cuanto a precio y condiciones. En algunos casos, las farmacias venden de manera clandestina la dosis que cada mujer solicita y el precio es generalmente elevado. Algunas mujeres, contaron con apoyo de familiares vinculados a profesionales de la salud que facilitaron acceso a receta médica para la compra del medicamento en farmacia. Otras buscaron fuera de fronteras (en Argentina o en Brasil), se conectaron con mujeres que habían abortado con ese método y que tuvieran medicación disponible o trataron de acceder a "bocas de venta" 
clandestinas del medicamento ${ }^{1}$. En todos los casos, la ruta de acceso a la medicación implicó un recorrido no exento de obstáculos, adicionando montos de ansiedad a la situación.

La mayoría de quienes abortaron con este método tenían alguna información previa. Sabían la forma de proceder con la medicación y las menos estaban al tanto de que podían sufrir intensos dolores o de los efectos colaterales que provoca su uso (náuseas, vómitos, diarrea). Pero hay otros factores del orden de lo subjetivo que se ponen en juego. Temor, miedo, angustia, ansiedad no sólo determinan el estado de ánimo de la mujer, sino que tienen un efecto directo en el cuerpo. El uso del misoprostol significa una experiencia corporal directa, que pone en marcha vivencias y fantasías concientes e inconscientes. Todo cambio que tiene lugar en el cuerpo, impone al psiquismo un proceso de elaboración mediante el cual se buscan representaciones mentales que le den significación y sentido a la experiencia, que al comienzo es sólo una experiencia sensible. El cuerpo subjetivo, la imagen corporal, las fantasías inconscientes del cuerpo, provienen de los datos que el cuerpo biológico envía, pero que están mediatizados por procesos de representación simbólica y significados colectivos de cada época o cultura. La información, aún comprendida desde el punto de vista cognitivo, no impidió que surgieran temores y fantasías de daño corporal o de muerte, asociados manifiestamente a la posibilidad de utilizar erróneamente las pastillas y/o no obtener el resultado esperado. Por esta razón y por el miedo a lo desconocido, fue que algunas mujeres prepararon el momento con extremo cuidado.

"Yo tenía todo preparado... pensaba que me iba a desangrar, que iba a necesitar toallas. Me puse las pastillas, fue el momento más tenso para mí. Tener que ponerme yo las pastillas y el miedo a no colocármelas bien. En ese momento tenía fantasías, porque en realidad era algo que le estaba haciendo a mi cuerpo

\footnotetext{
${ }^{1}$ En algunos contextos sociales críticos los puntos de venta clandestina de misoprostol también son puntos de venta de drogas de muy baja calidad, como por ejemplo pasta base.
} 
de lo que no tenía conocimiento total ni control. Eso me daba un poco de miedo" (Celia, 26, NEA).

"Lo hicimos de día porque pensamos que psicológicamente era más suave que hacerlo de noche. Me las puso mi compañero, yo tenía la idea de que no podía ponerlas hasta el fondo. Esos primeros diez minutos sentí que me iba a pasar algo. Yo lo miré a mi compañero 'me va a pasar algo' y él se re asustó, fue algo muy breve. Esa primer sensación fue fea. El miedo en sí, por lo físico, si me pasa algo" (Valeria, 21, NEA).

Los relatos del uso de misoprostol incluyeron fantasías de desangramiento, por el impacto que les produjo las hemorragias, la visión de los coágulos y los intensos dolores. Fue notoria la diferencia en el discurso entre las mujeres de diferentes niveles educativos. Las mujeres con nivel educativo bajo, lo hicieron con mayor crudeza, con menor grado de intelectualización que aquellas con nivel medio o alto. Para algunas, la ansiedad, el temor a colocarse mal las pastillas, el sentirse sola fueron factores que determinaron tener que repetir el procedimiento más de una vez, con lo que esto implicó en términos de costo emocional y económico. Más allá de las diferencias, todas las mujeres vivieron con intensidad, angustia y temor el momento de la práctica con misoprostol. A pesar de ello, varias mujeres manifestaron que acudirían nuevamente a este método, si necesitaran abortar.

"Mi único miedo era no expulsar y en ese caso tener que ir a la emergencia. No quería pasar esa situación, que me interrogaran [...]" (Valeria, 21, NEA).

\section{$\underline{\text { La atención hospitalaria post aborto }}$}

Si bien el estudio no se propuso indagar sobre la satisfacción de la atención hospitalaria, las mujeres espontáneamente hicieron referencia a sus valoraciones sobre el tipo de atención recibida a nivel institucional cuando debieron acudir para completar un aborto en curso. Tuvieron diferentes experiencias con el personal de salud. Algunas 
se sintieron respetadas y otras manifestaron haber sido objeto de valoraciones morales, enjuiciamientos y censuras.

“Una enfermera empezó a hablar conmigo y me dice 'si claro es difícil, pero es tu decisión', me decía 'es tu decisión, hay que respetarla. Yo no te voy a decir ni que a mi me parezca bien ni que me parezca mal, es tu decisión y ya está. No voy a poner mi punto de vista'. Y no ponía el punto de vista de ella” (Karina, 21, NEM).

"Yo llegué al hospital, me atendieron enseguida, me acostaron en una camilla, me sacaron los coágulos de sangre con los dedos y después con unos palos largos me limpiaban. El que mandaba dijo que me pusieran en una camilla con suero hasta que me trasladen a sala. Sin embargo, me mandaron a sentarme afuera. Yo me senté afuera y estaba hablando con dos mujeres que habían tenido familia y entonces me empecé a sentir mal, mal, mal. Le dije a mi hija que llamara a los médicos. Después no me acuerdo de más nada porque estuve quince minutos con perdida de conocimiento. Porque era demasiada la sangre que había perdido" (Alicia, 37, NEB).

\subsection{Los sentimientos en el post aborto}

Las mujeres entrevistadas, en su gran mayoría, refirieron que el sentimiento inmediato posterior fue el alivio por la culminación de un período previo marcado por la tensión y la inseguridad de practicarse un aborto clandestinamente y, a su vez, por haber dado término a una situación no deseada. Se pudo observar que a mayor convicción en la decisión, mayor fue la presencia de este sentimiento.

"Yo estaba muy firme en la decisión. Tengo dos hijos, cuando quise tener, los tuve. No me sentí culpable. Fue la mejor decisión y nunca me arrepentí de ello" (Fabiana, 41, NEA). 
"La primer sensación fue de alivio. No me siento culpable por eso tampoco. No tuve un dolor muy fuerte. Sentí que el duelo lo había hecho antes. Cuando ví que había expulsado y que evidentemente había salido todo, fue una sensación de alivio, porque realmente no lo quería tener. Yo quería terminar con esa situación. Por poder volver a mi vida, porque en esos quince días yo sentí que estaba en una realidad paralela, que el mundo funcionaba en un tiempo y yo iba en otro" (Valeria, 20, NEM).

Otros sentimientos fueron pena, tristeza, dolor psíquico, todas respuestas emocionales esperables frente una situación vital difícil. Haber pasado por la experiencia del aborto generó cuestionamientos acerca de sus propias vidas: posición frente a la maternidad, las estrategias de anticoncepción o sus relaciones afectivosexuales. Esta suerte de balance y/o autocrítica no estuvo exenta de dolor. Algunas mujeres habían "escuchado" que después de un aborto, la culpa y el malestar psicológico eran inevitables. Esas voces son producto de un imaginario social que naturaliza la culpa y la significa como un efecto sine qua non del aborto voluntario. Al sentir alivio, muchas mujeres experimentaron culpa por no haber sentido culpa.

Los sentimientos de culpa que emergieron no tenían una única génesis, sino que fueron producto de diferentes y complejos procesos subjetivos. En algunos casos, fueron la expresión de un conflicto entre el (los) deseo(s) y el mandato prohibidor, en otros la convicción de estar transgrediendo un idea o una norma. Pero los mandatos, los ideales, no surgen automáticamente en el sujeto por su sola condición humana, sino que son el resultado de un proceso de subjetivación que se realiza siempre con otros, desde los primeros momentos de la vida. $\mathrm{Y}$ son estos otros, quienes transmiten las significaciones acerca de lo permitido y lo prohibido, lo que es legítimo y lo que no lo es, según el momento histórico, la sociedad y la cultura en la que se constituyen como sujetos. En algunas mujeres, especialmente con nivel educativo bajo, se pudo observar nítidamente el conflicto entre las creencias religiosas y la decisión de no tener otro hijo, mostrando en forma descarnada la lucha interna entre intereses contrapuestos. Fueron 
ellas quienes parecieron ser más permeables al discurso hegemónico de la Iglesia Católica que condena al aborto al considerarlo un atentado a la vida, un asesinato, un pecado merecedor de castigo.

“Me preguntaba ‘¿qué fue lo que hice?’ ¿cómo sería el día de mañana, sería nena, sería varón?'. Eso fue lo primero que se me pasó por la cabeza. Si lo hubiera podido tener... pero que no lo quería tener. Es que la cabeza te empieza a dar vueltas. Porque no está permitido y porque te estás sacando algo que es tuyo. Para mí era algo malo, después... ya no. Hoy en día, ya no, tenía que asumirlo" (Sara, 24, NEB).

"Me siento dolida, porque hice eso que no se debería haber hecho. Mucha angustia, a veces estoy decaída. Pero la verdad que como mujer me sentí horrible. Ah! fue horrible, fue horrible hacerlo porque sabia que iba a matar un niño, que se me movía, ya con tres meses, tal vez estaba obsesionada, yo quiero mucho a mis hijos, ay! A los 16 fui madre por primera vez" (Josefina, 35, NEB).

“Después que lo hice le pedí a Dios que perdonara todo lo que había hecho, haberme sacado, haberme hecho el aborto. Porque me sentí con mucha culpa. Es por creencia, es porque yo creo en Dios, entonces claro para mi yo cometí un pecado, haberme hecho un aborto ¿verdad? porque sea lo que sea era una vida, ya estaba creciendo dentro de mí. Entonces por eso yo le decía a Dios que me perdonara por lo que había hecho, pero que él tenia que darse cuenta, que yo no lo podía traer al mundo porque no lo iba a poder mantener" (Alicia, 31 años, NEB).

Al significar al embrión como un niño, produce una representación de sí misma como mala, dañina y asesina, incapaz de satisfacer los ideales de bondad, de abnegación y cuidados que se supone una madre debe cumplir. 
El tiempo transcurrido entre el aborto y la realización de las entrevistas muestran diferencias en los relatos. Aquellas mujeres que habían abortado a menos de un año, se encontraban bajo el impacto emocional y presentaban signos de mayor ambivalencia, angustia y culpa. Se identificaron algunos elementos depresivos, tales como rabia, cambio de humor, angustia, sueños recurrentes, auto-reproches. Estos aspectos no son suficientes para tipificar una depresión, tomando en cuenta la escasa distancia temporal con el episodio y las condiciones en que se produjo el embarazo no deseado y el aborto. La mayor distancia temporal permitió la elaboración psíquica y en estas mujeres no se observaron evidencias de arrepentimiento, angustia y culpa. Pasado el tiempo, valoraban la decisión como acertada y no sentían que les hubiera dejado secuelas psicológicas o les hubiera significado una experiencia traumática, aunque la recordaran con detalles. Algunas de las entrevistadas, manifestaron no querer atravesar nuevamente por la experiencia y en palabras de una de ellas: "una cosa es hablar del aborto, otra es vivirlo".

“Acordarme, me acuerdo, porque es algo que pasó en mi vida y es algo espantoso que me pasó (haber quedado embarazada) que tuve la posibilidad y lo pude solucionar. No, por el aborto. Y lo pude solucionar, porque la verdad que hasta hoy en día, yo no me siento capacitada para ser madre y yo no pienso tener un hijo, si no estoy preparada no lo puedo tener" (Patricia, 26, NEM).

"No dudé. Realmente no quería., yo la decisión no la dudé nunca, no la dudé nunca. Ni aún hoy, cada vez que pienso, me digo: Qué acertada que estuve, ¡estuve bien!" (Graciela, 27, NEA).

No se encontraron evidencias que indiquen que la práctica del aborto haya producido efectos traumáticos, inhabilitantes y duraderos en el tiempo. La mayoría de las mujeres del estudio han continuado adelante con sus proyectos de vida y consideraron que el hecho no les afectó su vida sexual y/o afectiva hasta el presente. 


\subsection{Las mujeres frente al cambio legal sobre aborto}

Las mujeres entrevistadas expresaron opiniones favorables sobre la necesidad de un cambio legal sobre aborto en el país. Se pudieron identificar matices en los posicionamientos sobre este punto, especialmente referidos al tipo de despenalización que sería más adecuada. Estos matices y la construcción discursiva de los argumentos que los sostienen estuvieron vinculados, fundamentalmente, a la edad y el nivel educativo de las entrevistadas. La posición ideológica sobre el aborto, previa a la experiencia personal de estas mujeres, era heterogénea. En todos los casos, la experiencia vivida dejó sus marcas en sus actuales posiciones sobre el tema.

En cuanto a la ley que rige en el país, las mujeres expresaron distintos argumentos para fundamentar su opinión sobre la necesidad de cambiarla.

- La ley vigente legitima la hipocresía social: el estatuto legal legitima la hipocresía social en la medida que genera un circuito clandestino entorno a la práctica y no la desestimula, provocando inequidad en el acceso.

- La ley vigente desconoce el derecho y la libertad de decidir de las mujeres: las mujeres jóvenes y con nivel educativo medio y alto argumentaron su opinión en el reconocimiento y defensa del derecho de las mujeres a decidir sobre su propio cuerpo. Esta posición basada en la defensa de la libertad y la autonomía en la decisión, base de la teoría y la política feminista, parecería haber permeado el discurso de estas mujeres.

- La ley vigente expone a las mujeres a situaciones de riesgo de salud y de vida: las mujeres expresaron su preocupación sobre el aborto inseguro al cual están expuestas especialmente aquellas de menores recursos. Las mujeres dieron cuenta de su conocimiento sobre el uso de prácticas de riesgo: "había tomado té", "había ido a una casa de religión", "se metían agujas de tejer", "había usado ruda". 
- La ley vigente expone a las mujeres a situaciones de riesgo emocional: hicieron referencia al sufrimiento emocional que les generó la situación de clandestinidad. La vivencia fue de haber estado expuestas a situaciones de riesgo en su salud mental. El sufrimiento emocional que impone la ilegalidad y que acompañó todo el periplo, desde el momento que se tomó la decisión hasta su implementación, fue el riesgo más notable que refirieron estas mujeres. A esto se debe sumar el temor a la sanción legal, fuente de angustia pero no impedimento para que hubieran continuado con su decisión de abortar.

- La ley vigente favorece el lucro: el marco legal actual favorece el enriquecimiento de algunos en detrimento del sufrimiento de las mujeres. Varios relatos hicieron referencia a este punto, señalando que el sistema actual estimula los circuitos clandestinos que ofrecen prácticas de aborto de distinta calidad técnica, sin garantías de seguridad, de alto costo económico y sin responder por los resultados ni por las eventuales complicaciones.

- La ley vigente no evita que las mujeres aborten: las mujeres consideraron que la incidencia del aborto en la sociedad uruguaya es alta. La gran mayoría hizo alusión a mujeres conocidas que abortaron en distintas condiciones y circunstancias. Otras relataron la sorpresa que les generó encontrarse con un número significativo de mujeres en las salas de espera de las clínicas clandestinas y en la consulta hospitalaria. Algunas de ellas, tomaron conciencia en ese momento que había otras mujeres en su misma situación. Otras relataron que antes de vivir la experiencia, tenían la percepción de que el aborto era una práctica poco frecuente y que luego fueron tomando conciencia de la magnitud del aborto.

El denominador común se ubicó en la necesidad de despenalizar el aborto en el país. Pero, hubo matices respecto al alcance de esta despenalización. Algunas consideraron la importancia de reconocer el derecho a decidir de las mujeres y otras 
enfatizaron la necesidad de que la mujer tenga motivos que justifiquen su decisión. Para las primeras, la despenalización debía ser "controlada", "en función de los motivos para interrumpir" o en "función de cada situación en particular" e hicieron especial énfasis en dos circunstancias: alto número de hijos de las mujeres pobres y violación sexual. Esta opinión se sustentó en una visión controlista de la natalidad (cuestionando la maternidad de las mujeres pobres) y no en una visión de derechos humanos. Dos paradigmas diferentes y cuyo "enfrentamiento" - discursivo e ideológico - pervive en el imaginario social.

En un segundo grupo de opiniones, se encontraban aquellas que entendían que el aborto debía ser legal porque las mujeres tienen derecho a decidir sobre la continuidad o no de un embarazo. Fundamentaron que la decisión última es de la mujer, si bien lo deseable es que ésta sea compartida con el co-genitor. Subrayaron la importancia de poner limitaciones a este derecho, definir plazos en la edad gestacional (no más allá de las doce semanas) y/o limitar el número de abortos a los que una mujer pueda acceder.

Las mujeres enfatizaron la importancia de una ley que aborde integralmente la problemática, haciendo especial énfasis en el desarrollo de medidas educativas y preventivas respecto a la sexualidad y la reproducción. En general, hubo referencias difusas al debate político - parlamentario que atraviesa el país sobre este tópico. Se pudo observar una correlación entre el involucramiento en este debate y el tipo de vivencia sobre la experiencia propia. Sin establecer una tipología, parecería darse un patrón en el cual aquellas mujeres que participaron más activamente del debate social, analizaron la experiencia propia desde una mayor integralidad y con mejores posibilidades para procesar su situación personal. Por otro lado, aquellas que se mantuvieron aisladas de ese debate, tenían una perspectiva menos elaborada de su experiencia personal. 


\section{Discusión y conclusiones}

Los resultados de la investigación permiten conocer los efectos en la subjetividad de las mujeres que vivieron un proceso de aborto voluntario en un contexto de ilegalidad y penalización de la práctica como el caso uruguayo. No se encontraron evidencias que indiquen que el aborto haya producido efectos traumáticos, inhabilitantes y duraderos en el tiempo. La mayoría de las mujeres de este estudio que abortaron años atrás, han continuado adelante con sus proyectos de vida y consideraron que el hecho no les afectó su vida sexual y/o afectiva hasta el presente. Por tanto, no es posible afirmar, a partir de estos resultados que exista un síndrome traumático post evento, independientemente del nivel educativo y edad de la mujer.

Se puede afirmar que la ilegalidad y penalización genera altos montos de temor, incertidumbre y angustia. El contexto legal se transforma en texto que determina no sólo el acceso a un aborto médicamente seguro, sino también reacciones emocionales como las mencionadas.

Existe una directa relación entre origen del embarazo, condiciones para la toma de la decisión (recursos económicos, afectivos y sociales), acceso a un aborto seguro y sentimientos post - evento. En aquellas mujeres que tuvieron soporte afectivo y social para tomar la decisión, el sentimiento predominante posterior fue el alivio. A este sentimiento se asoció también pena, tristeza y dolor psíquico. Aquellas mujeres que se sentían ambivalentes frente al embarazo, vivieron una lucha interna entre un aspecto de sí mismas que no quería - o no podía- y otro que la condenaba por el no deseo de hijo. Al decidir abortar, lo hicieron desde la duda, lo que se tradujo luego en un intenso malestar subjetivo. Las relaciones de pareja estables no vieron afectadas su continuidad por la situación del aborto. Todas las mujeres entrevistadas continuaron con sus proyectos de vida, que incluyeron las opciones de tener o de no tener hijos.

El tiempo transcurrido entre el aborto y la realización de las entrevistas mostró diferencias en los relatos. Aquellas mujeres que interrumpieron su embarazo hacía menos de un año estaban en muchos casos, bajo el impacto emocional de todo el 
proceso y fue en ellas donde se observó mayor ambivalencia, angustia - y/o defensas contra la misma, fundamentalmente racionalizaciones - y culpa. Fue en estos casos, donde se pudieron observar algunos elementos depresivos: rabia, cambios de humor, angustia, sueños recurrentes sobre la experiencia, auto-reproches. Estos signos aislados no son suficientes para hablar de depresión, tomando en cuenta la escasa distancia temporal con el episodio.

Se observó una relación directa entre la significación previa acerca del aborto que tenían las mujeres y las repercusiones emocionales de todo el proceso. En aquellas mujeres que significaban el aborto como asesinato, la culpa fue el sentimiento posterior más intenso. Se constató que el arrepentimiento ante la decisión tomada fue acompañado de auto reproche y auto castigo. Estas significaciones se asocian a discursos que provienen desde lo social, vinculados fundamentalmente a las posiciones hegemónicas de las jerarquías eclesiales católicas y pentecostales. Esta situación se encontró solamente en las mujeres con nivel educativo bajo.

Ninguna de las mujeres del estudio vivió la experiencia de un aborto médicamente inseguro, por tanto no se constataron complicaciones severas desde el punto de vista biomédico. Sin embargo, el concepto de aborto inseguro adquirió otra significación, más allá de lo biomédico. Desde el punto de vista social, las mujeres dieron cuenta de su exposición a la censura y de las condiciones de silencio que acompañaron el proceso, independientemente de si hubieran contado o no con entornos familiares y sociales favorables y sostenedores.

El temor al rechazo socio -familiar y/o la sanción legal generó en algunas mujeres- como mecanismo de protección -no hablar de la experiencia-. El autosilenciamiento fue una forma de preservar la decisión, de no exposición al daño emocional y de evitar conflictos con seres queridos.

El acceso a información confiable, tanto para la toma de la decisión como para la efectivización del aborto, es una de las principales barreras que impone la clandestinidad de la práctica. Esta opera generando diques subjetivos y sociales para el acceso a la información que condiciona, a su vez, el acceso a prácticas más seguras. 
Aquellas mujeres que contaron con información y recursos de sostén afectivo, tuvieron mejores condiciones materiales y subjetivas para transitar y elaborar la experiencia vivida. Para otras, el temor al daño, la angustia por no saber o el tener información fragmentada y/o parcial, fueron algunos factores que acompañaron el proceso de toma de decisión, generando una cierta paralización frente a la situación. Esto estuvo vinculado con la falta o escasa disponibilidad de información sobre sexualidad y reproducción, las débiles condiciones sociales para el ejercicio de los derechos reproductivos, la fantasía sobre la invulnerabilidad personal que se traduciría en la frase "no me va a pasar a mi” y el contexto de ilegalidad que genera que la práctica del aborto continúe siendo predominantemente una práctica privada, a pesar de los avances notables que se pueden señalar en el campo del debate social en los últimos años.

El mundo de relaciones afectivas en las cuales cada mujer está inserta jugó un papel fundamental en el proceso de aborto y en las dimensiones subjetivas que se pusieron en juego. Si bien no necesariamente determinaron la decisión, sí incidieron en cómo se sintieron emocionalmente. En casi todos los casos, estas personas eran "sus iguales", es decir otras mujeres: madres, amigas, tías, abuelas, compañeras de trabajo, cuñadas, suegras. La actitud de esas "otras mujeres" fue de identificación con el malestar / sufrimiento / perplejidad que vivieron las entrevistadas, aunque sus respuestas no fueron homogéneas. Es de resaltar el rol que jugaron las madres de las mujeres, quienes fueron referencia, apoyo, compañía y respaldo en todo el proceso. Por su parte, el rol de los varones (co-genitores, padres, amigos) estuvo limitado, en muchas situaciones, a brindar apoyo económico para acceder al procedimiento.

Una de las diferencias en los relatos estuvo marcada por las vivencias que generó el tipo de procedimiento utilizado. Aquellas mujeres que abortaron en clínicas clandestinas mediante AMEU o legrado, refirieron que el mayor temor previo estuvo asociado al daño corporal, al riesgo de salud y de vida, incrementado por el uso de anestesia. Al largo periplo que las mujeres debieron transitar para acceder a las clínicas, se agregó otro caracterizado por el ambiente de secreto y riesgo que les reforzaba el estar cometiendo un delito punible. Aquellas que recurrieron al misoprostol se vieron 
enfrentadas a una experiencia corporal directa. Si bien la información recibida había sido comprendida por las mujeres, desde el punto de vista cognitivo, ello no impidió el surgimiento de temores y fantasías de daño corporal y/o de muerte, asociados a la posibilidad de equivocarse en la colocación de las pastillas y/o no obtener el resultado esperado. La vivencia corporal más recurrente fue la fantasía de estar desangrándose y los dolores abdominales intensos producidos por las contracciones.

Independientemente de las opiniones sobre los posibles caminos a recorrer para cambiar el marco legal sobre aborto en el país y los tipos de despenalización a implementar, el denominador común fue la necesidad de cambiar la ley vigente que tipifica el aborto como un delito siempre.

El estudio permite enfatizar que la información, la educación y el acceso universal a métodos anticonceptivos como componentes centrales de una estrategia de prevención de embarazos no deseados / no esperados, no son suficientes para impedir que éstos se produzcan. Cuando se trata de la condición humana, el diseño de las políticas públicas en salud reproductiva no puede soslayar las dimensiones subjetivas implicadas en la sexualidad y la reproducción. Existen razones más poderosas que la sanción jurídico - legal y moral, que determinan que una mujer opte por interrumpir un embarazo que no desea. Una mujer que aborta lo hace porque no encuentra otra salida ante un embarazo inoportuno o no deseado. Y al hacerlo decide arriesgarse a los efectos de la clandestinidad, poniendo en riesgo su salud (incluida la salud mental) y su vida. Esto denota la presencia de fuertes motivaciones subjetivas y una elección entre escasas alternativas.

Finalmente, el estudio presenta algunas limitaciones. Coincidimos con quienes sostienen que en tanto la búsqueda de las mujeres del perfil adecuado al estudio debe partir de la aceptación voluntaria de acceder a la entrevista, seguramente se establece un sesgo, pero ese sesgo es inevitable (Zamudio et al., 1998). Las mujeres que accedieron a relatar su experiencia de aborto, fueron probablemente aquellas que se sintieron en condiciones de hablar sobre ésta, tenían la necesidad de hacerlo y/o estaban preparadas 
- en alguna medida - para ello. A su vez, los hallazgos no pueden generalizarse a poblaciones con otro perfil que el incluido en el diseño muestral de nuestro estudio.

\section{Agradecimientos}

Las autoras desean agradecer las contribuciones para la implementación del estudio de todo el equipo de la Cátedra Libre en Salud Reproductiva, Sexualidad y Género de la Facultad de Psicología, así como al staff de MYSU, Mujer y Salud en Uruguay. Asimismo, queremos agradecer a las mujeres que, de manera voluntaria, ofrecieron sus relatos y su tiempo en las entrevistas. Finalmente, nuestro agradecimiento a la Comisión Sectorial de Investigación Científica de la Universidad de la República por apoyar financieramente este estudio. 


\section{BIBLIOGRAFIA}

- Adler, N. et.al. (1990): "Psychological Responses after Abortion". En American Association for the advancement of science. Science 6, april, vol. $248 \mathrm{n}^{\mathrm{o}}$. 4951, pp. 4144.

- Adler, N. (2000): “Abortion and the null hypothesis". En Archive General Psychiatry; $\mathrm{n}^{\circ} 57$, pp. 785-786.

- Amuchástegui, A. y Rivas, M. (2002): “Clandestine abortion in Mexico: a question of mental as well physical health". En Reproductive Health Matters, mayo, vol. 10, n. 19, pp. 95-102.

- American Psychological Association, Task Force on Mental Health and Abortion (2008): "Report of the Task Force on Mental Health and Abortion". Washington DC. [en línea] Disponible en: http://www.apa.org/pi/wpo/mental-health-abortion-report.pdf [09/04/2010].

- Carril, E. y López Gómez, A. (2008): Entre el alivio y el dolor. Mujeres, aborto voluntario y subjetividad. Montevideo: Ediciones Trilce.

- Cohen, S. (2006): “Abortion and Mental Health: Myths and Realities”. En Guttmacher Policy Review. Summer, vol. 9, no. 3, pp. 8-16.

- Coleman, P. et al. (2005): “The Psychology of Abortion: A Review and Suggestions for Future Research (Pandh, 4/05)". En Psychology and Health, april, 2005, vol. 20, n . 2, pp. 237-271, [en línea] Disponible en: http://www.physiciansforlife.org/content/view/792/26/ [14/12/06].

- Faúndes, A. y Barzelatto, J. (2005): El drama del aborto. En busca de un consenso. Editorial Tercer Mundo Editores del Grupo TM S.A.

- Fergusson, D; Horwood, L y Ridder, E. (2006): "Abortion in young women and subsequent mental health”. En Journal of Child Psychology and Psychiatry, jan, vol. 47, no. 1, pp. 16-24. 
- Fielding, S y Schaff, E. (2004): "Social Context and the Experience of a Sample of U.S. Women Taking RU-486 (mifepristone) for Early Abortion". En Qualitative Health Research, mayo, vol. 14, n. 5, pp. 612-627.

- Gómez Lavín, C. y Zapata García, R. (2005): “Categorización diagnóstica del síndrome post aborto”. En Actas Españolas de Psiquiatría, vol. 33, nº. 1, pp. 267-272.

- Knowles, J. (2007): “The emotional effects of induced abortion”, [en línea] Disponible en http://www.plannedparenthood.org/resources/research-papers/emotional-effectsinduced-abortion-6137.htm [14/03/07].

- Lacerda, I y Ribero, T. (2000): "Não vou esquecer nunca!: A experiencia feminina com o abortamento induzido". En Revista Latino-Amenfermagem, Riberao Preto, vol. 8, no. 6, pp. 50-58.

- Langer A. y Glas, A. (2003): "Embarazo no deseado y el aborto inseguro: su impacto sobre la salud en México”. En Gaceta Médica de México, julio-agosto, vol. 139, sup. 1, pp. 3-8.

- Lee, E. y Gilchrist, A. (1997): “Abortion Psychological Sequelae: the debate and the research. Psychology and reproductive choice”. En Conference EE.UU, [en línea] Disponible en: http://www.prochoiceforum.org.uk/psy_coun3.php [12/03/07).

- Major B. et al. (2009): “Abortion and Mental Health. Evaluating the evidence. En American Psychological Association, vol. 64, nº 9, pp. 863-890.

- Major, B. (2003): "Psychological implications of abortion - highly charged and rife with misleading research". En Canadian Medical Association, mayo, vol. 168, n. 10, pp. 1257.

- Major B. et al. (2000): "Psychological Responses of Women After First-Trimester Abortion”. En Archive of General Psychiatry, vol. 57, nº. 8, pp. 777-784.

- Nordal Broen, A. et al. (2004): "Psychological Impact on Women of Miscarriage Versus Induced Abortion: a Second Year Follow-up Study". En Psychosomatic Medicine, $\mathrm{n}^{\mathrm{o}}$. 66, pp. 265-271. 
- Nordal, A. et al. (2005a): "The course of mental health after miscarriage and induced abortion: a longitudinal, five-year follow-up study". En BMC Medicine, dec, $\mathrm{n}^{\mathrm{o}}$. 3, pp. 3-18.

- Nordal, A. et al. (2005b): "Reasons for induced abortion and their relation to women's emotional distress: a prospective, two-year follow-up study". En General Hospital Psychiatry, vol. 27, $\mathrm{n}^{\mathrm{o}}$. 1, pp. 36-43, [en línea] Disponible en: WwW.sciencedirect.com/science/journal/01638343 [12/03/2007].

- Organización Mundial de la Salud (2003): "El aborto sin riesgos. Guía técnica y de políticas para sistemas de salud", [en línea] Disponible en: http://whqlibdoc.who.int/publications/2003/9275324824_spa.pdf [12/03/2007].

- Osis, M. et al. (1996): "Dificuldades para obter informações da população de mulheres sobre aborto ilegal”. En Revista de Saúde Pública, oct, vol. 30, n. 5, pp. 444-51.

- Pope, L; Adler, N. y Taschann, J. (2001): "Postabortion Psychological Adjustment: Are Minors at Increased Risk?”. En Journal of adolescent health, vol. 29, nº 1, pp. 211.

- Reardon, D. C. et al. (2003): "Psychiatric admissions of low-income women following abortion and childbirth". En Canadian Medical Association Journal, may, vol. 168, $\mathrm{n}^{\circ}$. 10, pp. 1253-1256.

- Reardon, D. C. (1987): “Identifying High Risk Abortion Patientes”. En Paper Session of the Association for Interdisciplinary Research, [en línea] Disponible en: www.afterabortion.org/highriskk.html [12/03/2007].

- Stotland, N. (2003): “Abortion and Psychiatric Practice”. En Journal of Psychiatric Practice, march, vol. 9, nº. 2, pp. 139-149.

- Zamudio, L; Rubiano N.; Viveros, M. y Salcedo H. (1998): Problemas sensibles y estrategias metodológicas a propósito de investigaciones sobre aborto inducido. Bogotá: Centro de Investigaciones sobre Dinámica Social. Universidad Externado de Colombia. 